University of Nebraska - Lincoln

DigitalCommons@University of Nebraska - Lincoln

Development and characterization of the first infectious clone of alfalfa latent virus, a strain of Pea streak virus

Lev G. Nemchinov

USDA-ARS, lev.nemchinov@ars.usda.gov

Follow this and additional works at: https://digitalcommons.unl.edu/usdaarsfacpub

Nemchinov, Lev G., "Development and characterization of the first infectious clone of alfalfa latent virus, a strain of Pea streak virus" (2017). Publications from USDA-ARS / UNL Faculty. 1837.

https://digitalcommons.unl.edu/usdaarsfacpub/1837

This Article is brought to you for free and open access by the U.S. Department of Agriculture: Agricultural Research Service, Lincoln, Nebraska at DigitalCommons@University of Nebraska - Lincoln. It has been accepted for inclusion in Publications from USDA-ARS / UNL Faculty by an authorized administrator of DigitalCommons@University of Nebraska - Lincoln. 


\title{
Development and characterization of the first infectious clone of alfalfa latent virus, a strain of Pea streak virus
}

\author{
Lev G. Nemchinov
}

Accepted: 19 April 2017 This document is a U.S. government work and

(C) US Government 2017 is not subject to copyright in the United States.

\begin{abstract}
Alfalfa (Medicago sativa) is a natural host plant for many plant pathogens including fungi, bacteria, nematodes and viruses. Alfalfa latent virus (ALV) is strain of Pea streak virus, a member of the carlavirus group that occurs symptomlessly in alfalfa. The first complete genomic sequence of the ALV that was recently obtained in our laboratory showed that the virus differs substantially from other members of the genus Carlavirus. Here we report generation of infectious RNA transcripts from the constructed full-length viral cDNA clone as a proof that ALV nucleotide sequence is correct and as an initial step toward development of the ALV-based vector for gene silencing and expression of foreign proteins in alfalfa. This is the first report describing the development of a complete cDNA clone of the ALV strain of Pea streak virus and its infectivity in the diagnostic pea (Pisum sativum) and natural alfalfa hosts.
\end{abstract}

Keywords Alfalfa latent virus · Full-length infectious cDNA clone

Alfalfa (Medicago sativa) is a key forage crop for dairy producers in the US and in many countries of the world.

Electronic supplementary material The online version of this article (doi:10.1007/s10658-017-1237-2) contains supplementary material, which is available to authorized users.

L. G. Nemchinov $(\bowtie)$

Molecular Plant Pathology Laboratory, Beltsville Agricultural

Research Center, United States Department of Agriculture,

Agricultural Research Service, Beltsville, MD, USA

e-mail: lev.nemchinov@ars.usda.gov
It is an important component of sustainable agricultural systems because of its high yield, value for soil conservation, $\mathrm{N}_{2}$ fixation, crop rotation and wildlife habitat (Putnam et al. 2001). Alfalfa genomics is in the early stages of development primarily due to the complex genetic structure of this autotetraploid. Alfalfa is a natural host plant for many plant pathogens including fungi, bacteria, nematodes and viruses. One of the lowimpact viral pathogens infecting alfalfa is alfalfa latent virus (ALV). ALV was first described as a separate species and a new member of the carlavirus group that occurs symptomlessly in alfalfa (Veerisetty and Brakke 1977). It was later recognized to be a mild strain of Pea streak mosaic virus, genus Carlavirus, family Betaflexiviridae, due to their serological crossreactivity and particle length (Hampton 1981). In the United States, the virus is prevalent in Nebraska and Wisconsin (Veerisetty 1979). The first complete genomic sequence of the ALV strain of pea streak mosaic virus (PeSV) recently determined in our laboratory, showed that the virus differs substantially from other members of the genus Carlavirus (Nemchinov et al. 2015). Here we report generation of infectious RNA transcripts from the full-length viral cDNA as a proof that ALV nucleotide sequence is accurate and as the first step toward development of the ALV-based vector for gene silencing and expression of foreign proteins in alfalfa. Up to now, virus-induced gene silencing (VIGS), one of the most widely used tools in plant functional genomics, has not been applied toward alfalfa research due to lack of appropriate virus vector. Availability of specific VIGS vector would greatly facilitate studies of the key genes involved in various aspects of alfalfa development and adaptation to the environment. 
ALV-infected tissues used in this study were obtained from the ATCC under APHIS permit \# P526P-14-00733 (PV-264 isolate from Lancaster County, NE, USA). Total RNA was extracted with the TRIzol protocol (Thermo Fisher Scientific, Inc., Waltham, MA) according to the manufacturer's directions. The full-length ALV cDNA including 5' and 3' termini has been prepared as two fragments, KpnI-AgeI and AgeI-NotI, which were then assembled within the pUC57(Kan) plasmid by Medigen, Inc. (Frederick, MD) for a fee. All clones were sequenceverified. The constructs included $30 \mathrm{bp}$-long polyA tail at the $3^{\prime}$ terminus preceding NotI restriction site.

The full-length ALV cDNA in the pUC57 plasmid (pALV) contains the T7 RNA Polymerase promoter incorporated upstream of the $5^{\prime}$ end of the viral sequence (Fig. 1 and Supplementary Material Fig. S1). Plasmids were linearized with the NotI restriction enzyme and capped transcripts were generated in vitro from cDNA clones using Ambion's T7 mMessage Machine or Megascript T7 Transcription kits as advised by manufacturer (Thermo Fisher Scientific, Inc., Waltham, MA). Transcripts were rub-inoculated onto fully expanded leaves of two-week old Pisum sativum cv. Lincoln seedlings dusted with carborundum powder. Inoculated plants were kept in a containment greenhouse facility of the Molecular Plant Pathology Laboratory under 16-h day-light period.

Within three weeks after inoculation, pea plants developed chlorosis which became obvious one month after inoculation. Chlorotic leaves often had necrotic symptoms along their margins (Fig. 2). Western blot (WB) assay was performed with symptomatic leaves of $P$. sativum as described in Nemchinov and Natilla (2007). Membranes were probed with polyclonal antibodies to PeSV (AC Diagnostics, Inc., Fayetteville, AR). The serological test showed that pea plants are infected with the virus (Fig. 3a). Plant tissues of $P$. sativum were also examined by transmission electron microscopy (TEM). For TEM, leaves were homogenized in sterile water following by centrifugation of the extracts in a bench-top Eppendorf centrifuge for three minutes at $16.1 \mathrm{rcf}(13.2 \mathrm{krpm})$. Virus captured on the TEM grids was stained with $1 \%$ Phosphotungstate (PTA) solution. The grids were examined in the Hitachi H-7700 Electron Microscope at the Electron and Confocal Microscope Unit, Beltsville Agricultural Research Center. Flexuous virus particles with the modal length characteristic for ALV (636-640 nm) were observed in the extracts from the infected pea plants (as shown in Fig. $3 b$ for the purified preparation).

Following detection of the pathogen by WB and TEM, the virus was purified from the transcriptinoculated plants of $P$. sativum in order to obtain contaminant-free virus inoculum suitable for infection of alfalfa, since inoculation of alfalfa with crude extracts was not successful. TEM-verified infected plants of $P$. sativum cv. Lincoln were removed from soil and stored at $-20{ }^{\circ} \mathrm{C}$ until virus purification. The virus was purified from $\sim 85 \mathrm{~g}$ of whole pea plants (excluding roots) exactly as described in Veerisetty and Brakke (1978). Virus concentration estimated from the absorbance at $260 \mathrm{~nm}$ using NanoDrop ND 2000 spectrophotometer (Thermo Scientific) was $\sim 3.97 \mathrm{mg} / \mathrm{ml}$, assuming that extinction coefficient (E) for $0.1 \%(\mathrm{mg} / \mathrm{ml})$ solution at $260 \mathrm{~nm}=3.0$ (Veerisetty and Brakke 1978). Purified viral preparations examined by TEM contained flexuous filaments without any plant contaminants (Fig. 3b) and reacted positively with antiserum to PeSV in WB assays (not shown). To further confirm the identity of the virus, an RT-PCR was performed with the purified preparations using the following primers: $5^{\prime}$ CGATTGTGTCTCTGGTCATCTC 3' (forward primer, position 6400-6421) and 5' ATAAAGATGGCAGA

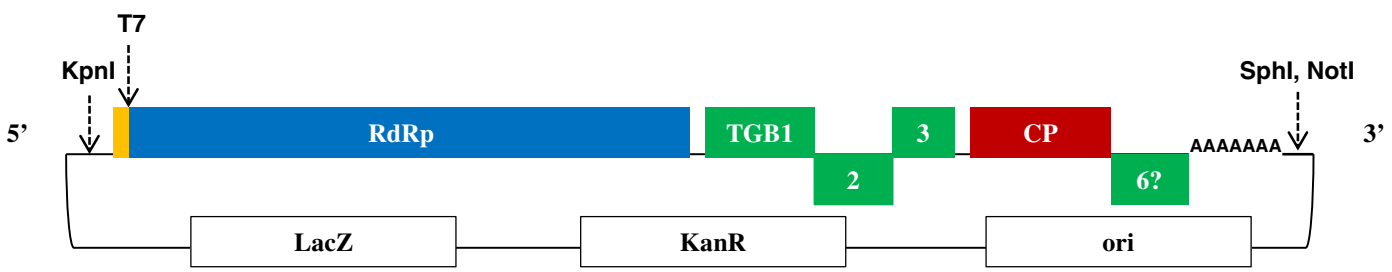

Fig. 1 Full-length ALV cDNA clone. T7: T7 RNA Polymerase promoter; RdRP: RNA-dependent RNA polymerase; TGB1, 2 and 3: triple gene block protein 1,2 and $3, \mathrm{CP}$ : capsid protein. 6 ?: putative nucleotide-binding protein (ORF 6). LacZ, KanR and ori from pUC57 plasmid backbone 
Fig. 2 Symptoms of alfalfa latent virus on transcript-inoculated plants of Pisum sativum cv. Lincoln. a Non-inoculated plant. b Plant inoculated with transcripts derived from the full-length cDNA clone of ALV
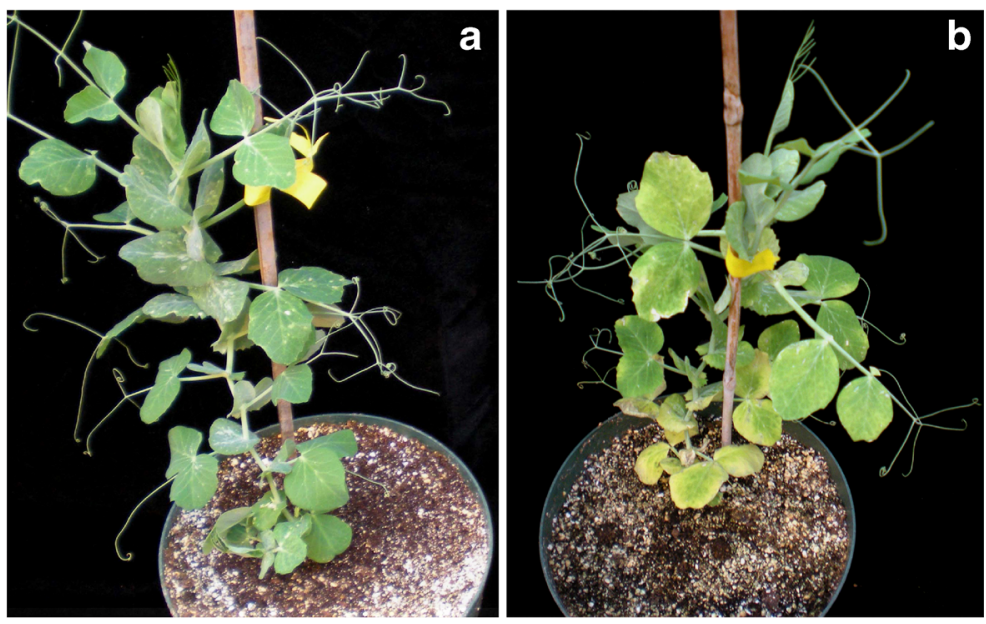

GCAACAGA 3' (reverse primer, position 7062-7083, GenBank accession number KP784454). The procedure,

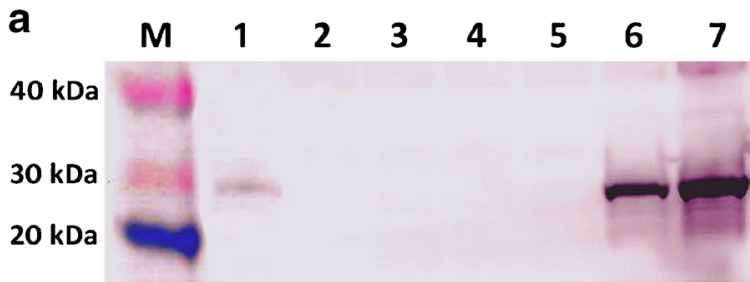

b

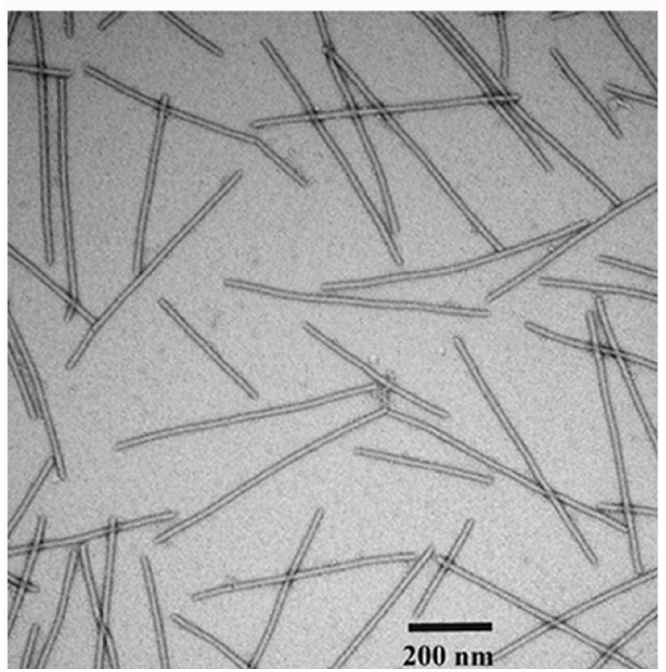

Fig. 3 a Western blot assay performed with symptomatic leaves of $P$. sativum inoculated with RNA transcripts. Membrane was probed with polyclonal antibodies to PeSV. M, Color Burst electrophoresis marker (Sigma-Aldrich). Lane 1, ALV positive control (1:10 dilution of the extracts from ALV-infected tissues obtained from ATCC); Lanes 2-5, uninfected $P$. sativum plants. Lanes 6 and 7: ALV-infected $P$. sativum plants. b ALV preparation purified from the infected plants of $P$. sativum cv. Lincoln. Scale bar represents $200 \mathrm{~nm}$ named simple-direct-tube RT-PCR (SDT-RT-PCR) was carried out essentially as described by Suehiro et al. (2005). It led to amplification of PCR product of the expected size $(684 \mathrm{bp})$ from all purified viral samples (Supplementary Material Fig. S2). Sequencing demonstrated that the amplified DNA segments corresponded to the targeted area of the ALV genome.

Two-week old alfalfa seedlings of Regen SY germplasm (Bingham 1991), acquired from the collections of National Plant Germplasm System were rubinoculated with purified viral preparations obtained from infected pea plants. Two weeks after inoculation, the virus was detected in non-inoculated upper leaves of alfalfa plants by WB with PeSV antiserum and by TEM (Supplementary Material Fig. S3). STD-RT-PCR performed with leaves collected from inoculated alfalfa plants was positive (not shown).

In conclusion, our results indicate that previously reported (Nemchinov et al. 2015) nucleotide sequence of the ALV was $99.9 \%$ correct. Only nine nucleotides in the reported sequence (GeneBank: KP784454) were different from the infectious clone (Supplementary Material Table S1). These nucleotides did not match between viral sequences determined by primer walking and Illumina RNA-seq and initial preference was given to the experimental RT-PCR approach (Nemchinov et al. 2015). RNA transcripts generated from the full-length cDNA clone of the virus were biologically functional and led to symptomatic infection in pea plants P. sativum cv. Lincoln. Virus particles possessing morphological and serological features of ALV were purified from the pea plants 
and used to successfully infect alfalfa germplasm Regen SY (Bingham 1991).

To the best of our knowledge, this is the first report describing the development of a complete cDNA clone of the ALV strain of PeSV and its infectivity in the diagnostic (Pisum sativum cv Lincoln) and natural (Medicago sativa) hosts. Generation of an infectious cDNA clone is an integral part of reverse genetic approach for any RNA virus; it is also a necessary tool for research on virus-host interactions and transient heterologous expression. Regarding this study, construction of the infectious ALV cDNA clone represents an initial step towards development of an ALV-based vector for gene silencing and expression of foreign proteins in alfalfa. To date, a breakthrough virus-induced gene silencing (VIGS) technology (Burch-Smith et al. 2004), available for other plant species has not been implemented in alfalfa research. An overall goal of the work is to introduce VIGS approach for functional genomics studies in alfalfa.

Acknowledgments This work would not have been possible without the skillful professional assistance of Peter Pushko of Medigen Inc. (Frederick, MD) and Joseph Mowery of the Electron and Confocal Microscope Unit, Beltsville Agricultural Research Center, Beltsville MD. The comments and suggestions of Rosemarie Hammond of Molecular Plant Pathology Laboratory and Dimitre Mollov of National Germplasm Recourses Laboratory, Beltsville Agricultural Research Center are greatly appreciated.

\section{Compliance with ethical standards}

Funding This work was supported by the United States Department of Agriculture, Agricultural Research Service.

Conflict of interest The author declares that he has no conflict of interest.
Human and animal rights This research does not include any animal and/or human trials.

Ethical approval The author bears all the ethical responsibilities of this manuscript.

\section{References}

Bingham, G. J. (1991). Registration of alfalfa hybrid Regen-SY germplasm for tissue culture and transformation research. Crop Science, 31, 1098-1098.

Burch-Smith, T. M., Anderson, J. C., Martin, G. B., \& DineshKumar, S. P. (2004). Applications and advantages of virusinduced gene silencing for gene function studies in plants. The Plant Journal, 39, 734-746.

Hampton, R. O. (1981). Evidence suggesting identity between alfalfa latent and pea streak viruses. Phytopathology, 71, 223.

Nemchinov, L. G., Shao, J., \& Postnikova, O. A. (2015). Complete genome sequence of the alfalfa latent virus. Genome Announcements, 3(2), e00250-15.

Nemchinov, L. G., \& Natilla, A. (2007). Transient expression of the ectodomain of matrix protein 2 (M2e) of avian influenza a virus in plants. Protein Expression and Purification, 56, 153159.

Putnam, D. H., Russelle, M., Orloff, S., Kuhn, J., Fitzhugh, L., Godfrey, L., Kiess, A., \& Long, R. (2001). Alfalfa, wildlife and the environment. In The importance and benefits of alfalfa in the 21st century. Novato: California Alfalfa and Forage Association.

Suehiro, N., Matsuda, K., Okuda, S., \& Natsuaki, T. (2005). A simplified method for obtaining plant viral RNA for RT-PCR. Journal of Virological Methods, 125, 67-73.

Veerisetty, V. (1979). Description of plant viruses. Alfalfa latent virus. http://www.dpvweb.net/dpv/showdpv. php?dpvno=211.

Veerisetty, V., \& Brakke, M. K. (1977). Alfalfa latent virus, a naturally occurring carlavirus in alfalfa. Phytopathology, 67, 1202-1206.

Veerisetty, V., \& Brakke, M. K. (1978). Purification of some legume carlaviruses. Phytopathology, 68, 59-64. 
Figure S1. Development and characterization of the first infectious clone of alfalfa latent virus, a strain of Pea streak virus. Lev G. Nemchinov. Molecular Plant Pathology Laboratory, Beltsville Agricultural Research Center, United States Department of Agriculture, Agricultural Research Service Beltsville, MD, USA. Email: lev.nemchinov@ars.usda.gov.

\section{Supplementary Figure S1}

\section{Full-length ALV cDNA clone in pUC57(Kan) vector (pALV)}

Small font: pUC57(Kan) sequence. Red fond: genomic sequence of ALV. Blue font: T7 promoter. Underlined: KpnI restriction site, ATG initiation codon, NotI and SphI restriction sites, respectively.

\begin{tabular}{|c|c|c|c|c|c|c|}
\hline & & & & & & \\
\hline 61 & & gat & $y \mathrm{ca}$ & & & \\
\hline 121 & ggcgggtg & & $\operatorname{atg}$ & & & \\
\hline 181 & & & & & & \\
\hline 241 & & & aactgttggg & agggcgatc & ggtgcgggcc & \\
\hline 301 & acgccagct & ggcgaaaggg & ggatgtgctg & aaggcgatt & aagttgggta & caccan \\
\hline 361 & gtc & acgacgttgt & aaacgacgg & att & gagctcggt & CCCTA \\
\hline 421 & & & & & & \\
\hline 481 & & & & & & \\
\hline 541 & & & CGC & & & \\
\hline 601 & & & & & & \\
\hline 661 & & & & & ACTCGC & \\
\hline 721 & & & & & & \\
\hline 781 & & & & & & $G$ \\
\hline 841 & & & & & & \\
\hline 901 & & & & & & \\
\hline 961 & & & & & & \\
\hline 021 & & & & & & \\
\hline 81 & & & & & & \\
\hline 41 & & & & & & \\
\hline 01 & & & & & & TG \\
\hline 61 & & & & & & \\
\hline 321 & & & & & & \\
\hline & & & & & & \\
\hline 41 & & & & & & \\
\hline 501 & & & & & CG & GC \\
\hline 61 & & & & & & CAA \\
\hline 21 & & & & & & \\
\hline 1681 & & & & & & \\
\hline & & & & & & \\
\hline & & & & & & \\
\hline & & & & & & \\
\hline & & & & & ACTTTGCGTA & GAGTATA \\
\hline 981 & & & & & & TTATAGT \\
\hline 041 & СТTCAАТTTG & & AAGG & GTG & GCCAGGTATG & TTTTGAGT \\
\hline & & & & & & \\
\hline & & & & & & \\
\hline & & & & & & \\
\hline
\end{tabular}


Figure S1. Development and characterization of the first infectious clone of alfalfa latent virus, a strain of Pea streak virus. Lev G. Nemchinov. Molecular Plant Pathology Laboratory, Beltsville Agricultural Research Center, United States Department of Agriculture, Agricultural Research Service Beltsville, MD, USA. Email: lev.nemchinov@ars.usda.gov.

2281 TTTTGAAAgG GCTGTCCACC ATATCGATTG CGAATGTGGC ATGAAGTTTG AATGCGgGAA 2341 AACTATGCTG GTTGATCATG CTAGAGTAAA TTTCATTGAT GATTTGGGCA ATAGGCAAGC 2401 GGTCTTCTTC AGTAAGTGCC AGGTTGATTA TAGGTATAAT GGTGGTGCGC ATGAAGCTGT 2461 TGAGTGGCCC GAGTGGTTGA ATATGCTCTT GGAATGCAAT GGCTTTGATC CGGAAGAGTA 2521 TGACTGCGTG CTAGTTCAAA TTTATTCAGG AGGTTCAAGT ATCGGTTTTC ATTCTGATGA 2581 TGAAGCGATT TTCCCTAAGG ACGGGAAAAT TCTGACCATG ACTACAGGCG GGGATGCGGA 2641 CTTTTCACTC CGATGCAAAG AAGGAGGCTT TACATTTCGT TTTGAGGCGA ACACCTACTT 2701 AATCATGCCT GAAGGTTGCC AAATCAGTCA TAAACATGCC GTAAGGGATT GTGATCATGG 2761 TCGTATAAGC TACACCTTTA GGCAATTAAA ACAATGCAGG GAGTATATGG AGCGTGAGTG 2821 TGAAGTTACT TCAAGCATCG ATCCATCAGA TGATGACCAA AGTGTAGAGA TCATTACCTT 2881 GCATGAGGTT GAGATCAAGC GTGGAAGAGA TGCGGATAAG CAGGCTTTAG AAGTGATTGA 2941 AGTGCCTGGT GATGGAAACT GCTTCTGGCA CTCATTGGGT TACTACTTTG GGCTAGAGGG 3001 TAGGTCGCTG AAGGAGATAA GTTTTTCGCG GTTAGAAGAG ATGGATTGGG ACTTGGAACC 3061 CCTCAAAAAA CAAATGAGTG GTTATGAGTT CTGCGAGTTG GAAAGTATTG TTGCTGCAGC 3121 AAGATTGCAC AGTGTGGAGA TAGAAATAAT TGATCACAAT AGTTCTATGG TTTGGGTGTT 3181 CACCCCAAAA AATAACTTAA GGCAGTATGC TAGGATGCAA CTTGTTGGAG AACATTATAG 3241 CCCCGTGATT CCAAAAGAGG TTTGTGTGCT AAGAGCTATA GCAGATTCTT TGGGAAAGTC 3301 TCTTCAAGAC ATCCATAGAT GTCTCACCAA AGCTGAGAAT CGCCACCTCC TTGAACTCGT 3361 TGAATCAGGT GAAGGTTTGG AAGTGTTTTT GATTGAGCCT TTCATGGTCT TATTTGGGAT 3421 CAGAGCAGTG ATCGATAATG ATGGGGACCT AATTGTTCTT AACGCAAAAG GCGCTCTTGA 3481 GAGATTCTTC CAAAATTACG GAGATCACCT CACCCACGTG AGTAAGGATA AAATTGTGGG 3541 TTTGGAGCGG TTTGGTGAGT TTGAGGGCCA GGGTGTTGAA AGGAACTCCC TCGCGGCGCT 3601 CAAGTCTGCA GGCACGGAAC TGATTTATGA AGCTAAGAAG GATAGGGCAG ACATCCTTTG 3661 TGAGAGTTTG CACTCCGGAT TAACTGGAGT AATCTCTTCA AAATTGTACA ATGATCAACC 3721 ATTGATCCAA AATCCCGGGC CGAACTCTAT TTGTAGGAGT TTAACTGTCA TTTTGGGGAC 3781 TTTCGGTGCG GGTAAGAGCA CTCTATTCAT TAAATTCCTT AAGGCGAATG AAGGCAAAAG 3841 AGTACATTTT GTTTCACCAA GAAAAGTTCT CGCAGAGGAA ATCAGATCCA ATATGGCACG 3901 AGCTCTTGGG TATGAGAGTG GACGAATGGG TAAGAGTGGG AAATTAAAGA GCAAAAATTG 3961 GTTTGTGCAC ACTTTTGAAG TTTTCCTACT TAAAATTCGG AGCCTAAAAG CTGAGGATTG 4021 TGTAGTCATG GATGAAGTGC AGCTTTTCCC ACCCGGTTAT TTGGATTGTA TGACTTACCA 4081 ACTGAATGGT AAGTGCGAGT TGATCGCTTT AGGTGATCCT GCGCAGAGTG ATTATGATAA 4141 TGAGAAAGAC AGGAATATCC TTTGCTGTTT AGGTTCGGAC ATAGAGCGGG CACTGGTAGG 4201 TAATGAGTAT GATTACACGA TTGGTTCATA CAGATTCCAA AACAGGAATT TTTTGAACAG 4261 ATTACCTTGC TCTTTTATGC ATCAGAGCTT GGAAATCGAT GAGCCTTACT TGATTTTTGA 4321 GGGTGTGGGC GAGTTCCTCA AGGTCTTCAC CGAGTACCGG TCAGTGGTGC TAGTGGCTGG 4381 TTTCACTGAA AAGAAGCTTG TGAGGGCTTA TTATGGTCAG GAATGCAGAG TTCTGACTTT 4441 TGGAGAGTCA ACAGGTCTAA CTTTCGATCG GGGTTTGATC ATTATAAGTG CTGATTCTTT 4501 AAAGGTCAAT GAGAAAAGGT GGGTCACAGC TTTATCAAGA TTCAGACAGA ATTTAGCCCT 4561 TTTGAACTTA ACTGGAGATA CTCTGGAGAA CCTTGTACTC CGGAAAGCCA ATTCAATGTT 4621 AGCTAACTTT CTGAAAGGCT CAAGCAAAGT CGAGAACCTC AAGGAAATTT TGCCTGGTGT 4681 ACCGAAGTTC AAACAAACTT TTATTGGAAA GGTAGGTAAA GATCAAGGCT TGGTTGAAGA 4741 AAAATTACAG GGTGATCCTT GGTTGAAAGG TGAATTATTT CTTGGCCAGA CTGAAGATGC 4801 AGAGGTGATC CAAATAAATG AATTCATGGA GCAGGATCAG TGGTTTAAAA CCCATTTGCC 4861 TCGCTGTGA ATGGAAGGGG TAAGAAGCAG GTGGCTGCAT TTGCTGCTTG AGAAAGAAGC 4921 AAGAGAATAC CGTTTCAGAG ATATGGTGAC CGAGCAATTC ACCGATGACC ATGATAGAGG 4981 TTGTGGTGAG AAATTGACCA ATGCGGCGGA GCGTTTTGAG GCGATCTACC CGAGGCACAG 5041 AGCTTCTGAT AATTTAACCT TCTTAATGGC CGTCAAGAAA AGATTGAGAT TCTCTAAGCC 5101 CCATATTGAG GAAGCCAAAC TGAATGAGGC CTTGCCGTAC GGCAAATTTC TATTGGATGA 5161 GTTCTTGCAG AAGATACCTC TGCGAGCAAC TCATAGACCT GACCTTATGG CTGAAGCTGT 5221 GCAGGATTTT GAGGAGAAGA AGAGAAGTAA AAGTGCTGCA ACTATAGAGA ATCATTCAGG 5281 CAGGTCCTGT AGGGATTGGT TGGCTGACGT TGGTTTAGTG TTTTCAAAAA GTCAGATTTG 5341 TACAAAATTT GATAACAGAT TTAGATGTGC CAAGGCTGCC CAGTCGATCG TTTGTTTCCA 
Figure S1. Development and characterization of the first infectious clone of alfalfa latent virus, a strain of Pea streak virus. Lev G. Nemchinov. Molecular Plant Pathology Laboratory, Beltsville Agricultural Research Center, United States Department of Agriculture, Agricultural Research Service Beltsville, MD, USA. Email: lev.nemchinov@ars.usda.gov.

5401 ACATTCTGTG TTATGCCGAT TTGCGCCCTA TATGAGATAC ATTGAAAAA TGCTAGGGGA 5461 GGCACTTAAA AAAACCAATT ACTATATCCA TTCTGGGAAG AGATTGGAAG ATCTGGATGA 5521 TTGGGTCGTC AAgGGTAATT TTTCAGgAAT TTGCACTGAA TCTGACTATG AAGCCTTTGA 5581 TGCTTCCCAG GATCATTGGA TCATGGCCTT TGAATTAACA CTGATGGAAC ATTTGGGGCT 5641 CCCAAAAGAT TTGATTGCGG ATTATAGATA CATTAAGACG CATTTGGGCT CCAAATTGGG 5701 AGCCTTTGCA ATCATGCGGT TCTCGgGgGA AGCTAGTACT TTCCTTTTTA ACACCATGGC 5761 CAACGCGCTT TTCACTTTCT TGAGATACGA CACTAGGGGT GATGAATTCA TATGCTTTGC 5821 TGGTGATGAT ATGTGTGCCT CAAAACATTT GAAGGTCAGC AACAAATTTG AGGATTTCTT 5881 GTCAAAACTT AAGTTGAAGG CTAAGGTTCA ATTTACCAAA AACCCGACAT TCTGTGGGTG 5941 GTCATTATTA CCTTTCGGAA TTTTCAAGAA GCCCCAATTG GTCTATGAAC GCATCTGCAT 6001 TGCTAGAGAA AAGAAGAACC TTCACAATTG CATAGATAAT TACGCAATTG AATTGTCGTA 6061 TGCTTATAAA AGAGGGGAGT TAGCAGTCAA TGCTATGAAT GAAGAAGAGA CTGATGCTTT 6121 TTACAATTGT GTCAGGGTCA TAATCAAAAG GAGCCACTTG CTGAAAAGTG ATGTCAAGGA 6181 TATCTTTCTC AATGCGAAAT CCCTCATGGA AAGTCTGAAT TAAGCTTAGA TCATCGCTAG 6241 TGTGGTTTGA TTAGATGGAT GTTTTCATAA AGAATTTAAT AGAGTGTGGT TTTCGTAGGA 6301 ATAAGGTTGT GTTTGACTTA CCCATCGTTG TTTTAGCTGT TCCGGGTGCG GGCAAAACTT 6361 CAAGCATTAG AAGGTTATTG CGCGAGGATT CTAGATTTGA AGCTTGGACT TTCGGAGTTC 6421 CTGATCATCA TAATTGTTCT GGTAGATTCA TTAAAGGGGT GACTGAGAGT TCTGAGCCTA 6481 ATCCTGATAA ATTCCTCATT GTGGATGAAT TTCAAAGAGg TGATTGGGAA AAATTCAAAC 6541 CTTTTGCTAT CTTCGGAGAC GTTGCGCAAT TAATGCTTAA GAATACCGCT TCCTTTGAAA 6601 GCGTGTTCTC CAAGTGTTCA TCCCATAGGG TTCCCTCATC CGTTGCTAAG TTGCTTCAGG 6661 AATTAGACTT CGAAATTACC AGTGAGCGTG AAGGTGTTTT GGAAATCAAA GATCTTCTTG 6721 GTTCTGAGCC GGAAGGTGTT GTGACCTGTT TTGAATCTGA AGTTTGTGAT TTGCTCGATT 6781 ACAACCAAGT TGATCATAAA AACCCCACTG AAGTCATCGG TTTGGAATTT CCGATTGTGT 6841 CTCTGGTCAT CTCCGGTAGA GCGGTTTTGG AGGTTCATAG AGCTGAATTC TACATTTGCT 6901 GCACAAGAGC GACAGATAAA TTAATAATCA TATCACCTGA GCCGGAGCAG TTCCATAGAG 6961 GATCCAATGC CATTGATAGC ACCTCCTAAT AATTCTAATT CTTATCTAGC TCTGGCTATC 7021 GGTGCTGGTT TTGCAATCAT CATCTTCACC CTTAGATCCA ATCAATTGCC TCACGTGGGT 7081 GATAACATTC ATTCATTGCC TCACGGAGGT TTTTACAGAG ACGgGACAAA GGTCATACAA 7141 TATAATTCCC CTGTGAGGAC GCCAAACAAC TGGTTCAAGG GACCTAACAA CATTCAGGCT 7201 TTAGCCCTGG TTTTGTTGGT TATTGGTCTG ATTCATGCGT CATCTGTAAA GATAAGTCGG 7261 GGATGCAGTT GTTCCAAGTA ATCCTAAGTG TGTTTTCCTG CTTTGCGGTT TTGTTGTGCT 7321 TGTATACTAT AGATTCGTTT TTAAATAATT CTGTGTGCCA GTGCACTGTG GTGTTAACTG 7381 GTGAAAGTGT CAAAATTGTC GgGTGTGAAT TTACAAGTGA ATTCATTGAG TACGCCAAAA 7441 CTTTAAAGGT GCAGGCAATT TGAgtatctt taagtttgca gagagtgtat tcgataaagA 7501 TGGCAGAGCA ACAGAAAGCA GCTGAGGAGA GGGCTCGCAT AGATGCGAAG TTGAAGCAGC 7561 AGCTGGAGCA GCAGAGTTCC AAACTGGGAG CTGAGAAAGA GTTTATTAAG GATGCTGAGG 7621 GTCTACAAAA ATCTTTACTT GAGCGGTTCC AGGCCTTACA AGACCTCACC GCTAAAAGCA 7681 TTGCAGGTTC TTCCATCGTT AATGGTGGTT GGGAAGCGGA TCGGAAAAGA CTCAAAGTTT 7741 CTGACAAAAT GAAGCTAGAT GGTTCCAACA TTTTCACAAG GCCAACATTG GACGATCTGC 7801 AGAAGCTGGG CTGGAATCCT GAGGCCAACC AGGTGGCTAC TGCTGAGAAT CTGGCTAAAA 7861 TCAGTGCGAA ATTGAAGGAA TTGGgGgTCC CGCCTGAACA GATGGCAAGg ACTTTTTGGg 7921 ATGTGGCTAT GTATTGTACT GCTGTGGGTG CTTCCAGGTA CACTAATCCA CAAGGGTCAA 7981 TTAACTATCC TGAAGGTTCA ATAACTAGAG ATGCGGTCTT TGGAGTCATT CGAGAGCAAT 8041 GCACTCTGAG GCAGGTTTGC AGGAGCTTCG CTCCTATCAT CTGGAACTAC ATGCATGTTA 8101 ATAATATGCC CCCAACTAAT TGGGCGGAAA AGGGTTTCAG CAATGATGTC AAATTTGCTG 8161 CTTTTGATTT CTTTGATTTC GTGGAGTGCC CAGCTTCAAT TCAACCGGCG GATGGTCTGA 8221 TTAGACGTCC TACATCTGAT GAGTATGTTG CGTTCAACAC TCACAAAAAG TGTGCCCTGG 8281 CCAGGGCTGA CAAGAATGGT CGTTATGCGA GTACTGATGC CTCTGTTACT GGAGGAATGT 8341 TCGGTTGTGG AGCCAAAGAG ACTTGGCGTA ATAATGCGTG CTGAATGTGT GTGCGGTTGC 8401 TAGTAAAACC TAAATAATGT ATAAGCTAGA ACCTATAAAA TCAAGTGTGT TTTAAAATAT 8461 TTTTAATGCT TGTAAAAAAA AAAAAAAAAA AAAAAAAAA AAAGCGGCCg catgcaagct 
Figure S1. Development and characterization of the first infectious clone of alfalfa latent virus, a strain of Pea streak virus. Lev G. Nemchinov. Molecular Plant Pathology Laboratory, Beltsville Agricultural Research Center, United States Department of Agriculture, Agricultural Research Service Beltsville, MD, USA. Email: lev.nemchinov@ars.usda.gov.

\begin{tabular}{|c|c|c|c|c|c|c|}
\hline & acaacataca & agccquaagc & ataaagtata & aagcctagag & acctaataa & \\
\hline 41 & tcacattaat & tgcattgcac & tcactgcccq & ctttccagtc & gaacctg & \\
\hline 8701 & tgcattaatg & aatcggccaa & cgcgggaa & gggcggttt & gtattggg & (5) \\
\hline 8761 & cttcctcgct & cactgactcg & gcgetcgg & teggct & $\operatorname{agcg}$ & \\
\hline 8821 & actcaaaggc & ggtaatacgg & ttatccacag & aatcagggga & taacgcagga & agaacatg \\
\hline 8881 & gagcaaaagg & ccagcaaaag & gccaggaacc & gtaaaaaggc & cgcgttgctg & gcgtttttcc \\
\hline 8941 & ataggctccg & ccccectgac & gagcatcaca & aaatcgacg & ctcaagtcag & aggtggcgaa \\
\hline 9001 & acccgacagg & actataaaga & accaggcgt & ttccecctgg & aagctccetc & gtgcgctctc \\
\hline 9061 & ctgttccgac & cctgccgctt & ccggatacc & $\operatorname{tgtccgcct} t$ & tctccettcg & gadgch \\
\hline 9121 & cgctttctca & tagctcacgc & gtaggtatc & tcagttcggt & gtaggtcgtt & yecou \\
\hline 9181 & tgggctgtgt & gcacgaaccc & cccgttcagc & ccgaccgctg & cgccttatcc & ggtaact \\
\hline 9241 & gtcttgagtc & caacccggta & agacacgact & tatcgccact & ggcagcagcc & actggtaaca \\
\hline 9301 & ggattagcag & agcgaggtat & gtaggcggtg & ctacagagtt & cttgaagtgg & tggcctaact \\
\hline 9361 & acggctacac & tagaagaaca & gtatttggta & tctgcgctct & gctgaagcca & gttaccttcg \\
\hline 9421 & gaaaaagagt & tggtagctct & tgatccggca & aаcaаaccac & cgctggtagc & ggtggttttt \\
\hline 9481 & ttgtttgcaa & gcagcagatt & acgcgcagaa & aaaaaggatc & tcaagaagat & cctttgatct \\
\hline 9541 & tttctacggg & gtctgacgct & cagtggaacg & aaaactcacg & ttaagggatt & ttggtcat \\
\hline 9601 & gattatcaaa & aaggatcttc & acctagatcc & ttttaaatta & aaaatgaagt & tttaaatcaa \\
\hline 9661 & gcccaatctg & aataatgtta & caaccaatta & accaattctg & attagaaaaa & ctcatcgagc \\
\hline 9721 & atcaaatgaa & actgcaattt & attcatatca & ggattatcaa & taccatattt & ttgaaaaagc \\
\hline 9781 & cgtttctgta & atgaaggaga & aaactcaccg & aggcagttcc & ataggatggc & aagatcctgg \\
\hline 9841 & tatcggtctg & cgattccgac & cgtccaaca & tcaatacaac & ctattaattt & cccotcgtca \\
\hline 9901 & aaataaggt & tatcaagtga & gaaatcacca & tgagtgacga & ctgaatccgg & tgagaatggc \\
\hline 9961 & aaaagtttat & gcattctett & ccagacttgt & tcaacaggcc & agccattacg & ctcgtcatca \\
\hline 021 & aatcactcg & catcaaccaa & ccgttattc & attcgtgatt & gcgcctgagc & gagacgaaat \\
\hline & acgcgatcgc & tgttaaaagg & acaattacaa & acaggaatcg & aatgcaaccg & gcgcagg \\
\hline & actgccagcg & catcaacaat & tettcacct & yaatcaggat & attcttctaa & tacctggaat \\
\hline 201 & gctgttttc & cggggatcgc & gtggtgagt & aaccatgcat & catcaggagt & acggataaaa \\
\hline 261 & tgcttgatgg & tcggaagagg & cataaattcc & gtcagccagt & ttagtctgac & catctcatct \\
\hline 0321 & gtaacatcat & tggcaacgct & acctttgcca & tgtttcagaa & acaactctgg & cgcatcgggc \\
\hline 381 & ttcccataca & agcgatagat & tgtcgcacct & gattgcccga & cattatcgcg & agcccattta \\
\hline 441 & tacccatata & aatcagcatc & atgttggaa & tttaatcgcg & gcctcgacgt & ttcccgttga \\
\hline 1001 & atatggctca & taacacccct & tgtattactg & tttatgtaag & cagacagttt & tattgttcat \\
\hline & gatgatatat & 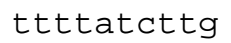 & & alcagagal & Llgagacac & gggccagagc \\
\hline & & & & & & \\
\hline
\end{tabular}


Development and characterization of the first infectious clone of alfalfa latent virus, a strain of Pea streak virus. Lev G. Nemchinov. Molecular Plant Pathology Laboratory, Beltsville Agricultural Research Center, United States Department of Agriculture, Agricultural Research Service Beltsville, MD, USA. Email: lev.nemchinov@ars.usda.gov.

\section{Supplementary Figure S2}

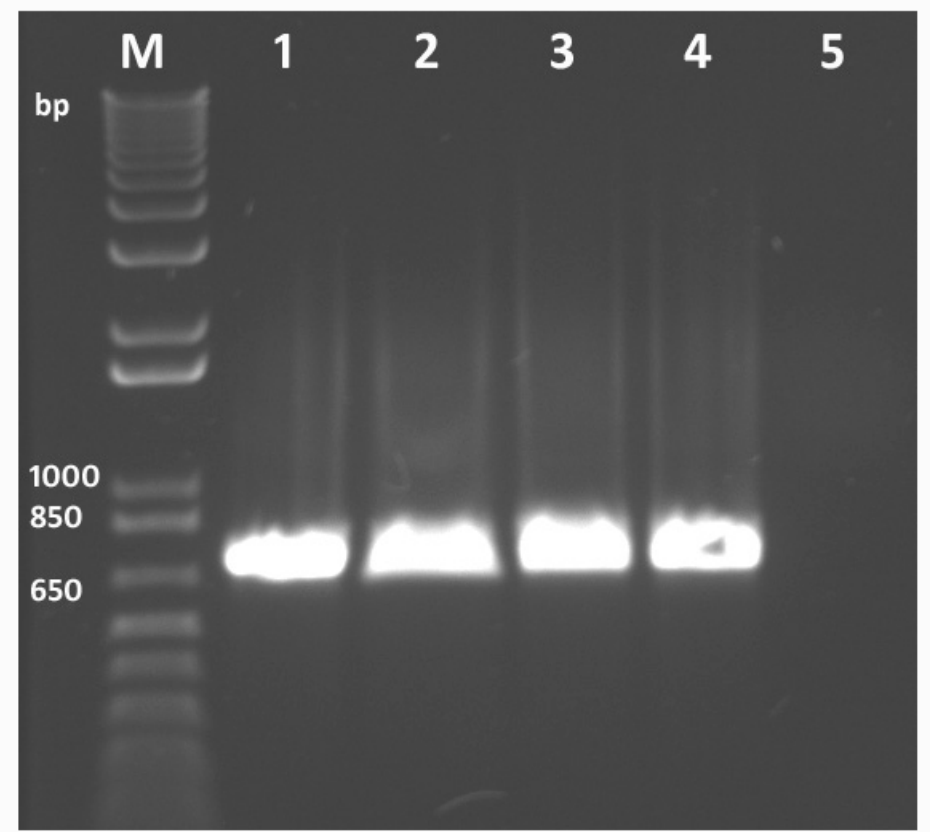

PCR products amplified from purified ALV preparations using STD-RT-PCR protocol

(Suehiro et al., 2005). The virus was purified from transcripts-inoculated plants. M, $1 \mathrm{~Kb}$

Plus DNA Ladder (Thermo Fisher Scientific). Lanes 1- 4: PCR products amplified from purified ALV preparations. Lane 5: water control. 
Development and characterization of the first infectious clone of alfalfa latent virus, a strain of Pea streak virus. Lev G. Nemchinov. Molecular Plant Pathology Laboratory, Beltsville Agricultural Research Center, United States Department of Agriculture, Agricultural Research Service Beltsville, MD, USA. Email: lev.nemchinov@ars.usda.gov.

\section{Supplementary Figure S3}

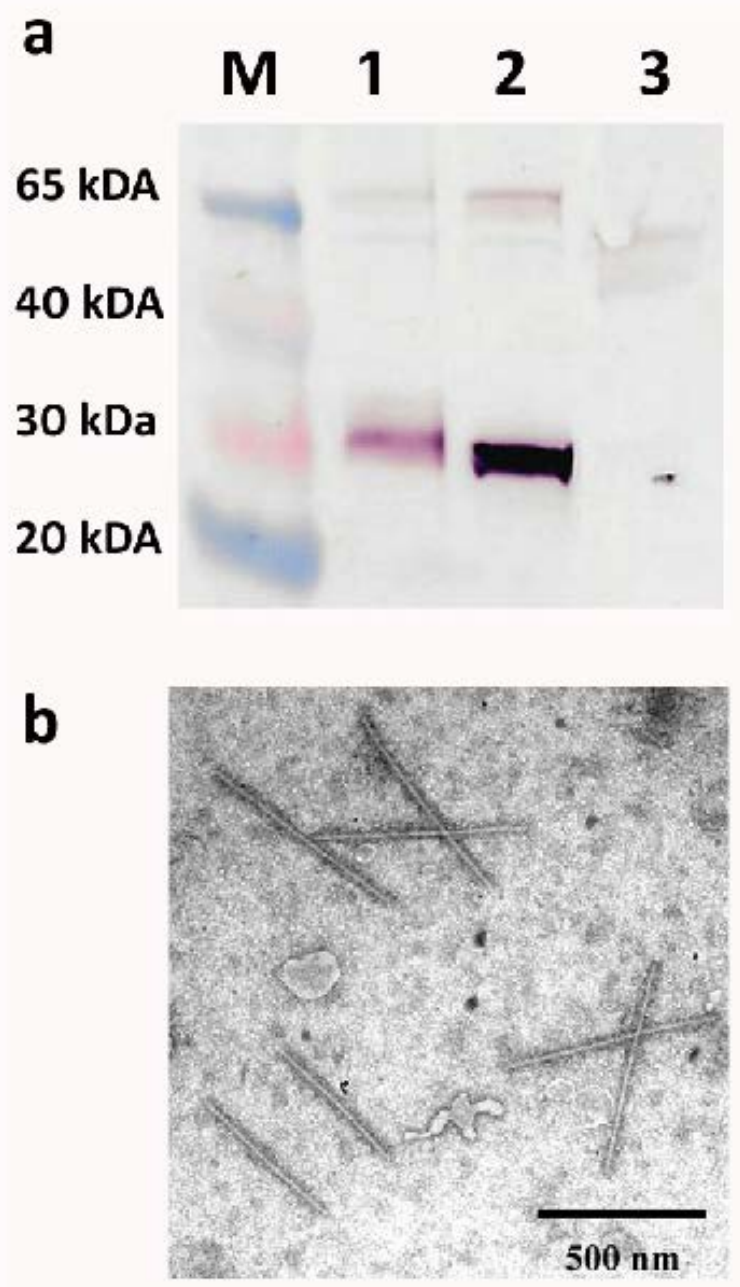

Serological and microscopic detection of ALV in crude extracts of alfalfa germplasm Regen SY inoculated with purified viral preparations from pea $P$. sativum cv. Lincoln. a Western blot assay using polyclonal antiserum to PeSV. M, Color Burst electrophoresis marker (Sigma-Aldrich). Lanes 1-3: ALV positive control, infected alfalfa plant Regen SY and uninfected alfalfa control, respectively. b TEM micrograph of ALV particles from infected alfalfa. Scale bar represents $500 \mathrm{~nm}$. 
Development and characterization of the first infectious clone of alfalfa latent virus, a strain of Pea streak virus. Lev G. Nemchinov. Molecular Plant Pathology Laboratory, Beltsville Agricultural Research Center, United States Department of Agriculture, Agricultural Research Service Beltsville, MD, USA. Email: lev.nemchinov@ars.usda.gov

\section{Supplementary Table S1}

Nucleotide differences between the reported ALV genome (GeneBank: KP784454) and the full-length infectious cDNA clone (this work)

\begin{tabular}{|c|c|}
\hline $\begin{array}{c}\text { GeneBank: KP784454 } \rightarrow \\
\text { infectious cDNA clone }\end{array}$ & Silent/missense \\
\hline $\mathrm{A} \rightarrow \mathrm{G}(\mathrm{nt} 2605)$ & GAA(Glu) $\rightarrow$ GAG(Glu), silent \\
\hline $\mathrm{C} \rightarrow \mathrm{T}(\mathrm{nt} 3439)$ & CTC(Leu) $\rightarrow$ CTT(Leu), silent \\
\hline $\mathrm{T} \rightarrow \mathrm{C}(\mathrm{nt} 3742)$ & GGT(Gly) $\rightarrow$ GGC(Gly), silent \\
\hline $\mathrm{C} \rightarrow \mathrm{A}(\mathrm{nt6530})$ & GGC(Gly) $\rightarrow$ GGA(Gly), silent \\
\hline $\mathrm{A} \rightarrow \mathrm{T}(\mathrm{nt} 6531)$ & \multirow{2}{*}{$\mathrm{ACA}(\mathrm{Thr}) \rightarrow \mathrm{TCC}(\mathrm{Ser})$, missense } \\
\hline$A \rightarrow C(n t 6533)$ & \\
\hline $\mathrm{C} \rightarrow \mathrm{A}(\mathrm{nt6534})$ & $\mathrm{CAT}($ His $) \rightarrow \mathrm{AAT}($ Asn $)$, missense \\
\hline $\mathrm{T} \rightarrow \mathrm{C}(\mathrm{nt6869})$ & TGT(Cys) $\rightarrow$ TGC(Cys), sillent \\
\hline $\mathrm{G} \rightarrow \mathrm{T}(\mathrm{nt} 6871)$ & TGT (Cys) $\rightarrow$ TTT(Phe), missense \\
\hline
\end{tabular}

